\title{
Microfluidic Bandage based on Diffusion Delivery
}

\author{
M. S. Seshasayee \\ Department of Chemical Engineering \\ National Institute of Technology Tiruchirappalli, Tamil Nadu, India \\ S. Gowtham \\ Department of Chemical Engineering \\ National Institute of Technology Tiruchirappalli, Tamil Nadu, India \\ V. Sudharsan \\ Department of Chemical Engineering \\ National Institute of Technology Tiruchirappalli, Tamil Nadu, India
}

\begin{abstract}
Over 400,000 people worldwide are living with Hemophilia A, a disease predominantly caused by deficiency of clotting factor VIII in the blood plasma. A localized application of the recombinant clotting factor VIII by integration with the contemporary bandage would help mitigate the damage caused by injuries in a hemophilia patient. The designed bandage would work on the principle of microfluidic diffusion delivery using a layer of membrane above which a microfluidic channel is present. The device was designed using a) Kinetic studies; b) Membrane Analysis; c) Finite element method. Hence an optimum design was achieved which provided maximum diffusion for minimum flow. The ease of application and the cheap manufacturing cost of the appliance, facilitates the large-scale production of this device at a low cost. This technique could further be extended to all drug delivery practices.
\end{abstract}

Keywords - Drug delivery, Microfluidic bandage, Bleeding disorder, PDMS membrane ,Clotting Factor

\section{INTRODUCTION}

Bleeding is the loss of blood due to damage to the blood vessel. Blood coagulation is a process that changes blood from a liquid into a gel forming a blood clot. It results in hemostasis cessation of bleeding. Blood coagulation is a result of 2 reactions - Platelet activation and the Coagulation cascade. These reactions are dependent on the concentration of platelets and 13 clotting factors present in blood plasma as specified by Davie and Earl ${ }^{[1]}$.

Bleeding disorders is the result of the inability of blood to form proper blood clots at the site of blood vessel injury. These are caused by defects in platelets or blood clotting factors.

Hemophilia A, a bleeding disorder that affects more than 400,000 people worldwide is caused due to genetic deficiency in clotting factor VIII. It leads to prolonged bleeding even from common injuries.

Depending on the severity of the disease, Gilbert, Rosendaal and Aledort ${ }^{[2]}$, classify Hemophilia A into three types. Individuals with less than $1 \%$ active factor are classified as having severe hemophilia, those with $1-5 \%$ active factor have moderate hemophilia, and those with mild hemophilia have between 5-40\% of normal levels of active clotting factor.

Individuals with severe hemophilia often require regular treatment to prevent internal bleeding especially in joints, muscle, digestive tract, brain. In addition to that, they require special first aid and subsequent treatment in the event of any injuries to prevent superficial bleeding.

\section{A. Traditional Methods}

One of the most common methods of treatment of hemophilia is replacement therapy where concentrates of clotting factor VIII is injected into the vein to compensate for the deficiency in the bloodstream. Replacement therapies are classified into two types on the frequency of their administration. Demand replacement therapies are provided to stop immediate bleeding whereas prophylactic therapies which are administered on a regular basis 
reduces the damage caused by bleeding even before first aid is received. However prophylactic therapies are more intensive and more expensive than demand therapies. According to Pier Mannuccio, DDAVP is conventionally used to treat mild Hemophilia $\mathrm{A}^{[3]}$. A literature research of 23 studies by Robson and Leung ${ }^{[4]}$ showed that $4.3 \%$ of the patients showed side effects. Hypertension and Seizures were the predominant complications that were noted ${ }^{[5-8]}$.

\section{B. Concept}

Microfluidic Bandage helps in the localized application of clotting factors by integration with a contemporary bandage. It consists of a layer of membrane above which a microfluidic channel is present. The user applies pressure on the bandage using a squeeze-chip which will cause the flow of fluid through the membrane into the wound site. The fluid contains a solution of recombinant factor VIII in blood plasma. Recombinant factor VIII is used to prevent infection associated with direct factor VIII transfusion. Bray and Gomperts proved that it doesn't have any side effects that is usually associated with desmopressin. ${ }^{[9]}$

\section{Membrane}

Polydimethylsiloxane (PDMS) Membrane is present above the microfluidic channel to enable the flow of liquid through it into the wound when the user applies pressure on the bandage using a squeeze-chip. It is a mineral-organic polymer (a structure containing carbon and silicon) of the siloxane family. PDMS is the most widely used siliconbased organic polymer and is particularly known for its unusual rheological (or flow) properties. PDMS membrane devices that are extremely flexible due to both the very low Young's modulus and the thickness of the membrane ${ }^{[10]}$. PDMS is optically clear, and, in general, inert, non-toxic, and non-flammable and anti-fouling properties. Due to the high permeability of PDMS, the membrane can be used to exchange gas or small molecules between two liquids or a gas and a liquid without direct contact. ${ }^{[11]}$ It also has a higher mass transfer coefficient than compared to other membranes ${ }^{[12]}$

\section{EXPERIMENT AND RESULTS}

In this present work, it is employed a microfluidic channel which is coupled with a PDMS membrane. Due to the immense pressure applied by the fluid on the membrane, diffusion delivery takes place. The required factor VIII concentration for a hemophilic patient was initially determined by studying the kinetics of blood coagulation ${ }^{\text {[16-17] }}$. The fig. shows that a steady state concentration of $1 \mu \mathrm{M}$ and flow velocity of $60 \mathrm{~mm} / \mathrm{s}$ is to be maintained using the delivery system proposed. Keeping the concentration of component factor VIII in blood at normal values as found in people not suffering from hemophilia promises steady localized application of the component for a clotting time of a healthy human being. This hence facilitates in reducing the clotting time of a hemophiliac patient.

\section{A. Kinetic Analysis}

According to Butenas and Saulius ${ }^{[18]}$, the concentration of Factor VIIIa in the blood of a healthy individual is around $1.22 \mathrm{nM}$. This concentration can be assumed to be uniform for all practical purposes. Assuming that the concentration of Factor VIIIa in a hemophilic individual is negligible, the desired Haemophilia A level increase can be calculated.

One IU (unit), as defined by the World Health Organization standard for blood coagulation factor VIII, human, is approximately equal to the level of factor VIII activity found in $1 \mathrm{~mL}$ of fresh pooled human plasma

Therefore the dose of Hemophilia Factor A in IU can be calculated by

$$
\text { Dose AHF (IU) }=\mathrm{W} \times \mathrm{D} \times 0.5
$$

where,

$\mathrm{W}=$ Weight of body in $\mathrm{kg}$

$\mathrm{D}=$ Desired AHF increase

The formula was used to find out the dose of Kogenate FS in terms of IU. Kogenate FS should be administered at the rate of $10 \mathrm{~mL}$ per minute at a concentration of 1 IU.Using the standard IU data for Kogenate FS for a body weight of $80 \mathrm{~kg}$, the concentration of the microfluid in the bandage was calculated to be $1 \mathrm{mM} .^{[19]}$

\section{B. Membrane Calculations}

The general equation of mass transfer in liquid boundary layer can be generally given by:

$$
\mathrm{J}_{\mathrm{i}}=\mathrm{K}_{\mathrm{l}} *\left(\mathrm{C}_{\mathrm{i}, \mathrm{b}}-\mathrm{C}_{\mathrm{i}, \mathrm{w}}\right)
$$

Where, 
$\mathrm{k}_{1}$ is the boundary layer mass transfer coefficient,

$\mathrm{C}_{\mathrm{i}, \mathrm{b}}$ the concentration of component I in liquid bulk,

$\mathrm{C}_{\mathrm{i}, \mathrm{w}}$ the concentration in liquid at the liquid-membrane interface

Based on the solution-diffusion model ${ }^{[21]}$, the flux of component i through the membrane (from membrane surface to permeate side) can be expressed as:

$$
\mathrm{J}_{\mathrm{i}}=\left(\mathrm{D}_{\mathrm{m}} / \mathrm{L}\right) * \mathrm{C}_{\mathrm{i}, \mathrm{m}}\left(1-\left(\mathrm{p}_{\mathrm{i}, \mathrm{d}} / \mathrm{p}_{\mathrm{c}}\right)\right)
$$

Where,

Dm is the diffusion coefficient in the membrane

$\mathrm{L}$ the membrane thickness

Thus, by varying the concentration inlets and taking the out stream pressures as normal blood pressure range, pressure inlet can be modeled. The required pressure drop to be exerted on the system comes out to be $11000 \mathrm{~Pa}$.

\section{Flow Calculations}

Hence a pressure of 11000 Pa must be exerted by the microfluidic system on the membrane. The microfluidic flow pattern was analyzed using the commercial software ANSYS 17.0 (licensed). The system is assumed to be in laminar, incompressible flow regime ${ }^{[22-24]}$ and is assumed to be a 3-D model. The pressure domain is as given in fig. (1). Since the flow is in microfluidic scale it is to be assumed as completely laminar. The viscosity of the fluid is assumed to be as of plasma as $1.5 \mathrm{cF}$. The pressure hence obtained (fig 1) on the membrane due to the flow is parameterized versus the inlet velocity. The flow velocity is analyzed in the range of $0-100 \mathrm{~mm} / \mathrm{s}$ and the respective pressure exerted on the membrane is calculated. A pressure above $11000 \mathrm{~Pa}$ is observed for velocities above $60 \mathrm{~mm} / \mathrm{s}$ as seen in fig. 2. The optimum inlet velocity which is to be supplied to the system is hence acquired.

To apply such a velocity to the system, micro-pumps, syringe pumps, squeeze chips can be used. The specific advantage with the latter is that squeeze chips pave way for in-situ application for the device. Velocities upward of $600 \mu \mathrm{L} / \mathrm{min}$ can be achieved using a squeeze-chip ${ }^{[25]}$. Hence making it possible and practical for in-situ applications.

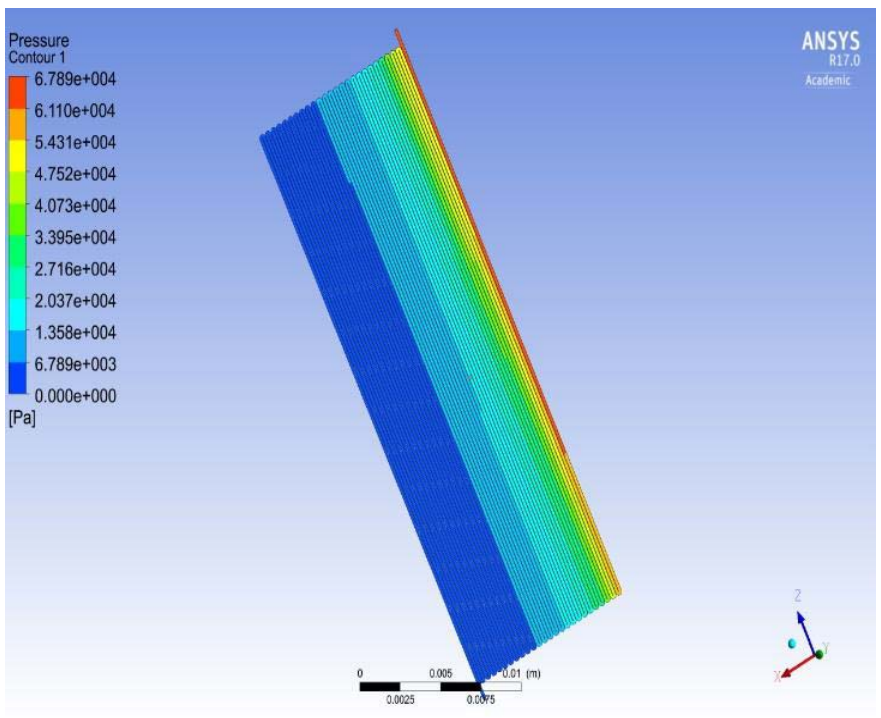

Figure 1. Pressure distribution at $\mathrm{v}=75 \mathrm{~mm} / \mathrm{s}$ 


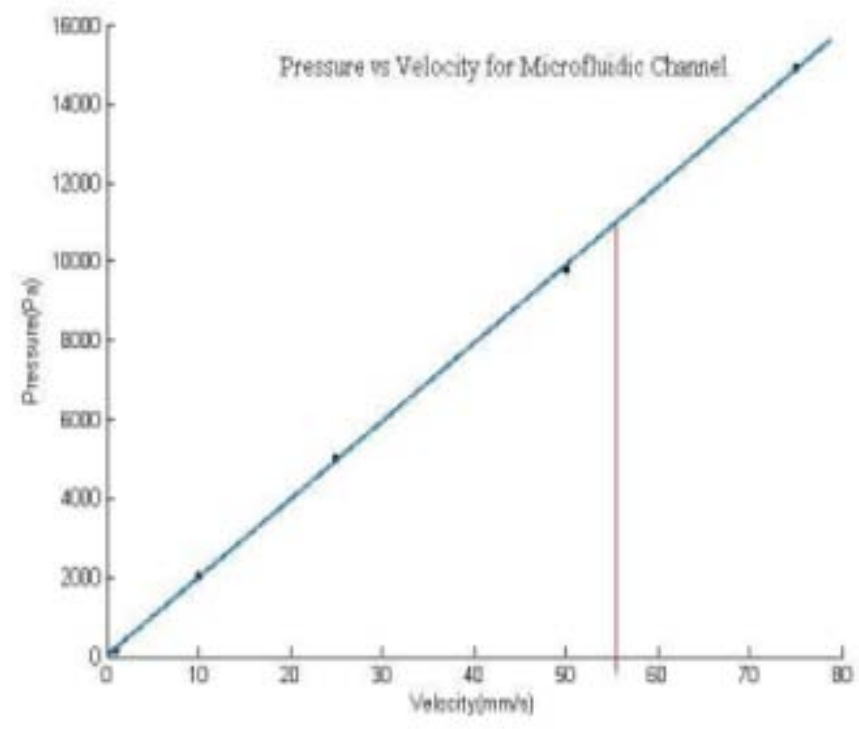

Figure 2. Pressure distribution for various velocities

\section{DESIGN OF THE DEVICE}

\section{A. Membrane configuration and preparation}

Membranes were configured in such a way that its thickness is minimum so that blood permeates through the membrane easily. The thickness of the membrane was found to be around $0.000003 \mathrm{~m}$ (300 microns).

The study is aimed to provide a low-pollution and high-efficiency preparation method using water as a solvent. ${ }^{[29]}$ The PDMS membrane is generally prepared on a wafer which will be used in a spin coater to realize the PDMS membrane. Before use, the wafer has to be cleaned. The wafer is first cleaned with acetone, then dried with air and then kept in an oven at $120^{\prime} \mathrm{C}$ for 5 minutes.

The PDMS membranes were then prepared by solution casting method as given by Sun, $\mathrm{De}, \mathrm{Li}$, and $\mathrm{Xu}^{[25]}$. For the preparation of unfilled PDMS membrane, a casting solution containing $17 \mathrm{wt} \%$ PDMS was prepared by dissolving PDMS, crosslinker (ethyl silicate) and curing agent (dibutyltindilaurate) in the solvent (n-heptane) with a ratio of 10:1:0.5 (in weight). The solution was subjected to homogenization by magnetic stirring of 3 hours; after the preparation of PDMS casting solution, it was poured onto the surface of thenon-woven fabric for 15 seconds. The PDMS coated flat sheet membrane was dried in the sterile room at room temperature for 24 hours, and then the crosslinked unfilled PDMS flat sheet composite membrane was prepared. The composite membranes with skin layers of variable thickness could be achieved by controlling the concentration of PDMS solution or the coating amount. 


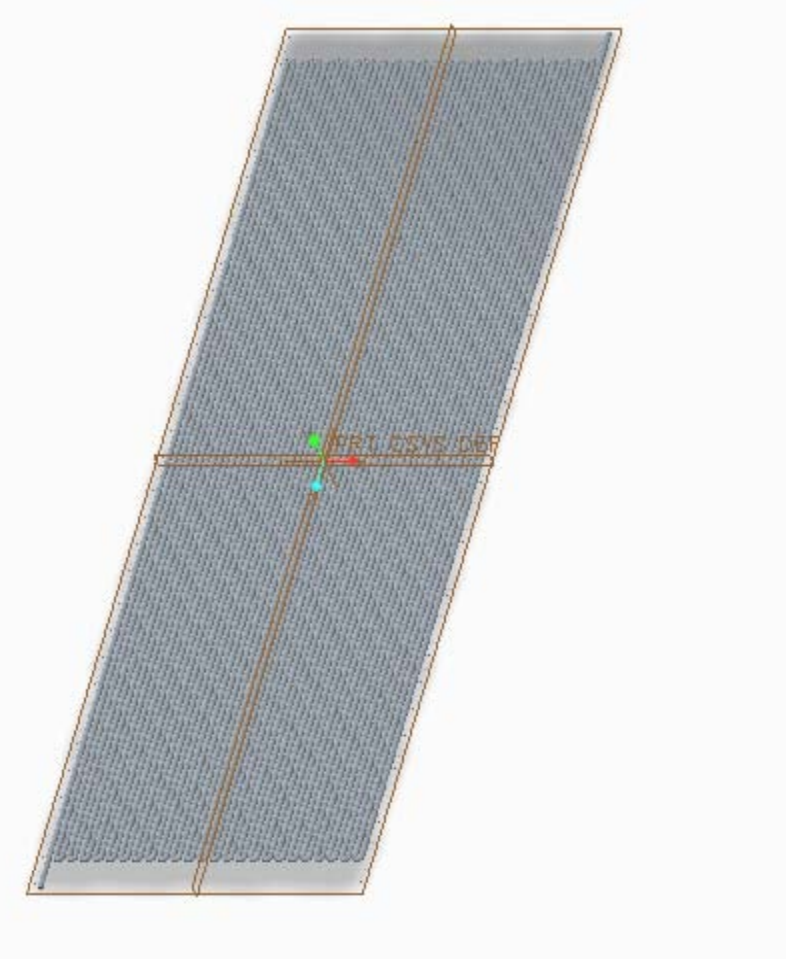

Figure 3. The optimum microfluidic channel design

\section{B. Micro-channel configuration}

The given microchannel is a semi-cylindrical in nature with a diameter of $10 \mu \mathrm{m}$. It is made of silicon material coated with the hydrophobic membrane to repulse the plasma and ensure smooth flow of liquid. The same is manufactured using lithography techniques ${ }^{[26-27]}$. There are over 20 such channels winding over to provide maximum pressure to the channel flow as shown in fig 3 . The number of channels and the diameter was decided such that the pressure through the system is maximized. On decreasing the diameter of the system, due to constraints because of the viscosity of the fluid against the walls of the system, the net pressure generated decreases. However, increasing the diameter would also result in the same issue. Hence an optimum size is achieved accordingly after parameterizing the diameter through simulations.

\section{Squeeze chip specifications}

The squeeze chip ${ }^{[25]}$ is a device which supplies the required flow rate to the channel. Since a flow velocity of 60$70 \mathrm{~mm} / \mathrm{s}$ is to be generated, a series of squeeze chips and variations in flow channel diameters is employed.

\section{Squeeze chip specifications}

The plaster is such that it gives support to the membrane and microfluidic channel beneath. It shouldn't be poisonous and shouldn't cause deleterious effects to the user. The adhesive bandage designed by Lauritzen(1985) using acrylate serves the purpose at hand ${ }^{[31]}$.

\section{IV.CONCLUSION}

The microfluidic bandage designed can serve as an in situ application for wounded hemophilic patients.The given device is found to provide sufficient delivery of the deficient factor VIIIa to the patients. In addition, it is easy to operate as it uses a basic push mechanism. The proposed microfluidic bandage that has been designed theoretically is to be further fabricated and experimentally analyzed. The devices could further be extended on to treat other bleeding disorders such as Hemophilia B and Von Willebrand disease.Therefore, microfluidic bandage with PDMS 
membrane can attract great interest for application in many important drug delivery practices.Further work is also going on to study the working of this device in intravenous applications

\section{REFERENCES}

[1] Davie, Earl W., and Kazuo Fujikawa. "Basic mechanisms in blood coagulation." Annual review of biochemistry 44, no. 1 (1975): $799-829$.

[2] White, Gilbert C., Frits Rosendaal, Louis M. Aledort, J. M. Luscher, Chantal Rothschild, and Jørgen Ingerslev. "Definitions in hemophilia." THROMBOSIS AND HAEMOSTASIS-STUTTGART- 85, no. 3 (2001): 560-560.

[3] Mannucci, Pier Mannuccio. "Desmopressin (DDAVP) in the treatment of bleeding disorders: the first 20 years." Blood 90, no. 7 (1997): 2515-2521.

[4] Robson, W. L., and A. K. Leung. "Side effects and complications of treatment with desmopressin for enuresis." Journal of the national medical association 86, no. 10 (1994): 775.

[5] Andersson, K-E., and B. Arner. "Effects of DDAVP, a synthetic analogue of vasopressin, in patients with cranial diabetes insipidus." Journal of Internal Medicine 192, no. 1-6 (1972): 21-27.

[6] de la FUENTE, B. E. A. T. R. I. Z., Carol K. Kasper, Frederick R. Rickles, and Leon W. Hoyer. "Response of patients with mild and moderate hemophilia A and von Willebrand's disease to treatment with desmopressin." Annals of internal medicine 103, no. 1 (1985): 6-14.

[7] Siew, Dou-Anne, Joy Mangel, Lori Laudenbach, Sheila Schembri, and Leonard Minuk. "Desmopressin responsiveness at a capped dose of $15 \mu \mathrm{g}$ in type 1 von Willebrand disease and mild hemophilia A." Blood Coagulation \& Fibrinolysis 25, no. 8 (2014): $820-823$.

[8] Mannucci, P. M., V. Vicente, I. Alberca, E. Sacchi, G. Longo, A. S. Harris, and A. Lindquist. "Intravenous and subcutaneous administration of desmopressin (DDAVP) to hemophiliacs: pharmacokinetics and factor VIII responses." Thrombosis and haemostasis 58, no. 4 (1987): 1037-1039.

[9] Bray, G. L., E. D. Gomperts, S. Courter, R. Gruppo, E. M. Gordon, M. Manco-Johnson, A. Shapiro, E. Scheibel, and M. Lee. "A multicenter study of recombinant factor VIII (recombinate): safety, efficacy, and inhibitor risk in previously untreated patients with hemophilia A. The Recombinate Study Group." Blood 83, no. 9 (1994): 2428-2435.

[10] Thangawng, Abel L., Rodney S. Ruoff, Melody A. Swartz, and Matthew R. Glucksberg. "An ultra-thin PDMS membrane as a bio/micronano interface: fabrication and characterization." Biomedical microdevices 9, no. 4 (2007): 587-595.

[11] Firpo, G., E. Angeli, L. Repetto, and U. Valbusa. "Permeability thickness dependence of polydimethylsiloxane (PDMS) membranes." Journal of Membrane Science 481 (2015):

[12] Mayer, Philipp, Ulrich Karlson, Peter S. Christensen, Anders R. Johnsen, and Stefan Trapp. "Quantifying the effect of medium composition on the diffusive mass transfer of hydrophobic organic chemicals through unstirred boundary layers." Environmental science \& technology 39, no. 16 (2005): 6123-6129

[13] Scherson, Daniel A., and Melvyn I. Burk. "Oxygen producing bandage." U.S. Patent 5,855,570, issued January 5, 1999.

[14] Scherson, Daniel A., and Melvyn I. Burk. "Oxygen producing bandage and method." U.S. Patent 5,578,022, issued November 26, 1996.

[15] Lo, Joe F., Martin Brennan, Zameer Merchant, Lin Chen, Shujuan Guo, David T. Eddington, and Luisa A. DiPietro. "Microfluidic wound bandage: localized oxygen modulation of collagen maturation." Wound Repair and Regeneration 21, no. 2 (2013): $226-234$.

[16] Mester, Zoltan, Joseph Powers, and Samuel Paolucci. "Mathematical Modeling of the Kinetics of the Extrinsic Blood Coagulation System with Stoichiometric Anticoagulants." (2007).

[17] Chatterjee, Manash S., William S. Denney, Huiyan Jing, and Scott L. Diamond. "Systems biology of coagulation initiation: kinetics of thrombin generation in resting and activated human blood." PLoS Comput Biol 6, no. 9 (2010): e1000950.

[18] 1-8.Butenas, Saulius, Behnaz Parhami-Seren, Anetta Undas, David N. Fass, and Kenneth G. Mann. "The “normal” factor VIII concentration in plasma." Thrombosis research 126, no. 2 (2010): 119-123.

[19] Henrard, Séverine, Niko Speybroeck, and Cedric Hermans. "Impact of being underweight or overweight on factor VIII dosing in hemophilia A patients." haematologica 98, no. 9 (2013): 1481-1486.

[20] Zhang, Yuhua, Masatoshi Ishida, Yutaka Kazoe, Yohei Sato, and Norihisa Miki. "Water-vapor permeability control of PDMS by the dispersion of collagen powder." IEEJ Transactions on Electrical and Electronic Engineering 4, no. 3 (2009): $442-449$.

[21] Li, Lei, Zeyi Xiao, Shujuan Tan, Liang Pu, and Zhibing Zhang. "Composite PDMS membrane with high flux for the separation of organics from water by pervaporation." Journal of Membrane Science 243, no. 1 (2004): 177-187.

[22] Low, Wan Shi, Nahrizul Adib Kadri, and Wan Abu Bakar bin Wan Abas. "Computational fluid dynamics modelling of microfluidic channel for dielectrophoretic biomems application." The Scientific World Journal 2014 (2014).

[23] Hashim, Uda, PN A. Diyana, and Tijjani Adam. "Numerical simulation of microfluidic devices." In Semiconductor Electronics (ICSE), 2012 10th IEEE International Conference on, pp. 26-29. IEEE, 2012.

[24] Olesen, Laurits Højgaard. "Computational fluid dynamics in microfluidic systems." Master's thesis, Technical University of Denmark (2003).

[25] Li, Wentao, Tao Chen, Zitian Chen, Peng Fei, Zhilong Yu, Yuhong Pang, and Yanyi Huang. "Squeeze-chip: a finger-controlled microfluidic flow network device and its application to biochemical assays." Lab on a chip 12, no. 9 (2012): 1587-1590. Y. Yorozu, M. Hirano, K. Oka, and Y. Tagawa, “Electron spectroscopy studies on magneto-optical media and plastic substrate interface,” IEEE Transl. J. Magn. Japan, vol. 2, pp. 740-741, August 1987 [Digests 9th Annual Conf. Magnetics Japan, p. 301, 1982].

[26] Sun, De, Bing-Bing Li, and Zhen-Liang Xu. "Preparatio n and characterization of poly (dimethylsiloxane)-polytetrafluoroethylene (PDMSPTFE) composite membrane for pervaporation of chloroform from aqueous solution." Korean Journal of Chemical Engineering 30, no. 11 (2013): 2059-2067.

[27] Pal, Prem, and Kazuo Sato. "Various shapes of silicon freestanding microfluidic channels and microstructures in one-step lithography." Journal of Micromechanics and Microengineering 19, no. 5 (2009): 055003. 
[28] Han, Xiaowei, Xiaowei Liu, Li Tian, He Zhang, and Zhi-Gang Mao. "A Non-Photolithography Fabrication for a Microfluidic Chip Based on PMMA Polymer." Machines 3, no. 2 (2015): 107-122.

[29] Wu, Yong, Zeyi Xiao, Weixing Huang, and Yuehua Zhong. "Mass transfer in pervaporation of active fermentation broth with a composite PDMS membrane." Separation and purification technology 42, no. 1 (2005):

[30] Li, Shufeng, Fan Qin, Peiyong Qin, M. Nazmul Karim, and Tianwei Tan. "Preparation of PDMS membrane using water as solvent for pervaporation separation of butanol-water mixture." Green Chemistry 15, no. 8 (2013): 2180-2190.

[31] Lauritzen, Nels J. "Adhesive bandage and package." U.S. Patent 4,549,653, issued October 29, 1985. 